\section{Extragalactic astronomy}

Galaxies and the Universe. (Stars and Stellar Systems: Compendium of Astronomy and Astrophysics, Vol. 9.) Edited by A. Sandage, M. Sandage and Jerome Kristian. Pp. xxii +818 . (University of Chicago: Chicago and London, 1976.) £30; \$45.00.

IT is 50 years since Edwin Hubble discovered Cepheid variable stars in the M31 galaxy, an epochal step in surveying the extragalactic universe, 21 years since this series was conceived, and 14 years since Allan Sandage commenced the editorial work for this book. The result is a book which is a product of the 1960s and which contains articles completed between 1965 and 1973, with a mean epoch of 1970 . Is a book six years out-of-date worth $£ 30$ ?

Fortunately that is an improper question: the 19 chapters are written by people with the highest reputation, each contributing in a field in which he or she is an expert. As Sandage rightly says in his preface, one remarkable feature is the enduring quality of the chapters, which are definitely not out-of-date, although some (for example, quasars, clusters, radio emission) are certainly not up-to-date. The editorial brief was to provide a compendium of essential facts about the Universe, and this policy has ensured that emphemeral theoretical playthings and tentative observations of implausibilities have been kept to the irreducible minimum. Not an easy task in extragalactic astronomy!

Galaxies and the Universe provides a review of well-established knowledge on the observed properties of galaxies and quasars. Individual chapters discuss the contents, masses, magnitudes, colours, sizes, energy distributions, and radio properties of galaxies and quasars. There are also extensive contributions on the dynamics and structure of galaxies. Observational cosmology is dealt with in sections on the distance scale of the universe, the distribution of galaxies, and an impressive review by the editor entitled "The Redshift".

The style is that of a review adressed to an informed reader, and there are very extensive bibliographies. Over 1,000 astronomers feature in the Name Index, and 1,200 objects in the Galaxy Index: these statistics give an impression of the ambitious scope of the work.

Galaxies and the Universe stands as a fitting synoptic monument to a heroic age in the quest to explore the Universe. Here are the results of a golden half-century that successively established the existence of island galaxies beyond the Milky Way, the redshift, radio galaxies, the cosmic background radiation, and quasi-stellar objects. The nature and history of the Universe on the largest scales is now yielding to the methods of science, and the main thrust of astronomical research for the rest of this century will be among the galaxies. Here in broad outline is a competent summary of what we know about those remote objects, and an indication of what we need to find out. It is an absolutely invaluable work of reference for anyone working in the field and an essential purchase for libraries. It is probably the most significant astronomical book published this year.

Simon Mitton

Simon Mitton is Secretary of the Institute of Astronomy, University of Cambridge, and Editor of the Quarterly Journal of the Royal Astronomical Society.

\section{Amphibian physiology}

Physiology of the Amphibia. Vol. 3. Edited by Brian Lofts. Pp. xiv +644 . (Academic: New York and London, July 1976.) $\$ 58.50$; $£ 35.70$.

BeCAuSE of their phylogenetic position and varying degrees of adaptation to life in two media the Amphibia are a group with which comparative physiologists are rightly very much concerned. In this, the presumably final, volume of Physiology of the Amphibia, the somewhat scattered literature in several fields is brought together and critically reviewed. The completed series now constitutes a source of information which should be indispensable to all herpetologists who are physiologically inclined. The timescale for the appearance of the various parts has, however, been unduly extended and it is a measure of the advances which have been made in some aspects of the subject in the intervening twelve years that there are articles on metamorphosis in both volumes one and three. Regrettably the prices of these same two volumes are an even more striking testament to the pace of inflation.

The present volume covers integumentary aspects such as colour change (J. T. Bagnara), moulting (L. L. Larsen) and resistance to dessication (E. Elkan); cell culture (K. A. Rafferty) and immunity mechanisms (E. L. Cooper); general neuroanatomy (A. Oksche and $\mathbf{M}$. Ueck); more specialised articles on the visual (D. Ingle) and auditory (R. C. Capranica) systems; and finally metamorphosis, with special emphasis on its biological and eco- logical aspects (M. H. I. Dodd and J. M. Dodd). The declared editorial policy has been to group related topics in the same volume but perhaps inevitably the final one seems to be the most heterogeneous, although there is more interrelation between some of the chapters than might at first appear. For instance it is helpful to have the account of the neuroendocrine systems alongside the discussion of the role of neurosecretion in metamorphosis.

The authors of the articles on cell culture and immunity mechanisms both stress the advantages of using amphibian material for general work in these fields; if this should lead to stocks of Amphibia being maintained in more laboratories, then the homily on amphibian husbandry in the chapter on pathology (E. Elkan) is very timely. At least as far as the more terrestrial species are concerned it must be realised that Amphibia in captivity are pathological specimens unless the conditions closely reproduce those found in the natural habitat.

The book is well produced and printing errors are rare, and then only of a minor nature.

C. L. Smith

Dr Smith is a Lecturer in the Department of Zoology at the University of Liverpool, UK.

\section{Are you involved in cancer research?}

\section{Do you read}

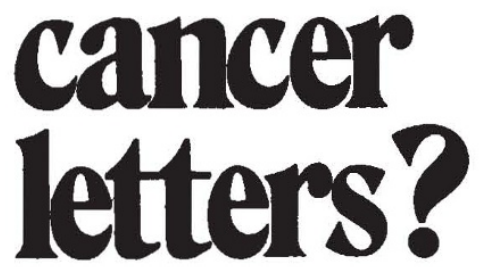

\section{Co-managing editors \\ Dr. P. Shubik \\ and Dr. D. Clayson}

The international journal which specialises in the rapid publication of short reports on all aspects of cancer research.

For a subscription and/or a specimen copy, please write to the Promotion Manager, ELSEVIER/NORTH-HOLLAND BIOMEDICAL PRESS B.V., P.O. Box 1527, Amsterdam, The Netherlands.
0399 